PROCEEDINGS OF THE

AMERICAN MATHEMATICAL SOCIETY

Volume 131, Number 9 , Pages 2883-2893

S 0002-9939(02)06931-9

Article electronically published on December 30, 2002

\title{
IRREGULAR GABOR FRAMES AND THEIR STABILITY
}

\author{
WENCHANG SUN AND XINGWEI ZHOU
}

(Communicated by David R. Larson)

\begin{abstract}
In this paper we give sufficient conditions for irregular Gabor systems to be frames. We show that for a large class of window functions, every relatively uniformly discrete sequence in $\mathbb{R}^{2}$ with sufficiently high density will generate a Gabor frame. Explicit frame bounds are given. We also study the stability of irregular Gabor frames and show that every Gabor frame with arbitrary time-frequency parameters is stable if the window function is nice enough. Explicit stability bounds are given.
\end{abstract}

\section{INTRODUCTION}

Fix some $g \in L^{2}(\mathbb{R})$; then the windowed Fourier transform is defined by

$$
\left(F_{g} f\right)(t, \omega)=\int_{-\infty}^{+\infty} f(x) \overline{g(x-t)} e^{-i x \omega} d x, \quad \forall f \in L^{2}(\mathbb{R}),
$$

where $g$ is called a window function. If $\left\{e^{i b_{n} x} g\left(x-a_{n}\right): n \in \mathbb{Z}\right\}$ forms a frame for $L^{2}(\mathbb{R})$, which is called a Gabor frame or a Weyl-Heisenberg frame, then every $f \in L^{2}(\mathbb{R})$ is uniquely determined by its discrete windowed Fourier transform $\left(F_{g} f\right)\left(a_{n}, b_{n}\right)$.

Since Gabor [19] proposed a signal representation with windowed Fourier transform, Gabor systems have had a fundamental impact on the development of modern time-frequency analysis and have been widely used in communication theory, quantum mechanics, and many other fields. For a collection of papers related to Gabor frames and their applications to signal and image processing, we refer to the monograph [18.

An important problem in practice is to determine conditions on the window function $g$ and sampling points $\left\{\left(a_{n}, b_{n}\right)\right\}$ which imply that a Gabor system is a frame. For the regular case, i.e., $\left\{\left(a_{n}, b_{n}\right): n \in \mathbb{Z}\right\}=\{(n a, m b): n, m \in \mathbb{Z}\}$, many results including necessary and sufficient conditions for $\left\{e^{i m b x} g(x-n a): m, n \in \mathbb{Z}\right\}$ to be a frame are established. For details, see [4, 8, 9, 10, 11, 12, 17] 23] 27].

Received by the editors August 29, 2001 and, in revised form, March 2, 2002 and April 11, 2002 .

2000 Mathematics Subject Classification. Primary 41A58, 42C15, 42C40.

Key words and phrases. Gabor frames, Weyl-Heisenberg frames, stability.

This work was supported by the National Natural Science Foundation of China (Grant Nos. 10171050 and 10201014), the Mathematical Tianyuan Foundation (Grant No. TY10126007), the Research Fund for the Doctoral Program of Higher Education, and the Liuhui Center for Applied Mathematics. 
For the irregular case, Christensen, Deng and Heil (see [9]) proved that for $\left\{e^{i b_{n} x} g\left(x-a_{n}\right): n \in \mathbb{Z}\right\}$ to be a frame, it is necessary that $\left\{\left(a_{n}, b_{n}\right)\right\}$ is relatively uniformly discrete and is of lower Beurling density no less than $\frac{1}{2 \pi}$. Also, Ramanathan and Steger [26] proved that the density must be exactly $\frac{1}{2 \pi}$ in order to get an exact frame.

For sufficient conditions, however, very few results are known. Feichtinger and Gröchenig [16] proved that if $F_{g} g$ is integrable on $\mathbb{R}^{2}$, then there exists an open set $U \subset \mathbb{R}^{2}$ such that $\left\{e^{i b_{n} x} g\left(x-a_{n}\right): n \in \mathbb{Z}\right\}$ is a frame for $L^{2}(\mathbb{R})$ for every separated set $\left\{\left(a_{n}, b_{n}\right): n \in \mathbb{Z}\right\} \subset \mathbb{R}^{2}$ with $\bigcup_{n \in \mathbb{Z}}\left[\left(a_{n}, b_{n}\right)+U\right]=\mathbb{R}^{2}$. Unfortunately the Feichtinger-Gröchenig theory does not give much information on how to choose an appropriate set $U$. However, it has many attractive features, e.g., it actually delivers discrete expansions in a very large class of Banach spaces 5, 16. Other results concerning specific window functions or sampling points can be found in [3, 24, 28.

For a band-limited window function $g$, Gröchenig [21] gave sufficient conditions which ensure $\left\{e^{i \lambda_{m} x} g\left(x-\mu_{m, n}\right): m, n \in \mathbb{Z}\right\}$ to be a frame with explicit frame bounds. In this case, the sampling points $\left(\mu_{m, n}, \lambda_{m}\right)$ lie in parallel lines.

In [30], we proved that $\left\{e^{i \lambda_{m, n} x} g\left(x-\mu_{m, n}\right): m, n \in \mathbb{Z}\right\}$ is a frame for certain $g$ if $\left(\mu_{m, n}, \lambda_{m, n}\right) \in[n a,(n+1) a] \times[m b,(m+1) b]$ and $a, b$ are small enough. We also gave explicit frame bounds since they are necessary for implementing the frame algorithm.

In this paper, we show that if $g(x), x g(x) \in H^{1}$, then $\left\{e^{i b_{n} x} g\left(x-a_{n}\right): n \in \mathbb{Z}\right\}$ is a frame for $L^{2}(\mathbb{R})$ for every relatively uniformly discrete and $(a, b)$-dense sequence $\left\{\left(a_{n}, b_{n}\right): n \in \mathbb{Z}\right\}$ if $a$ and $b$ are small enough. All constants including frame bounds are determined explicitly.

The stability of frames is needed in practice. Given a frame $\left\{e^{i b_{n} x} g\left(x-a_{n}\right): n \in\right.$ $\mathbb{Z}\}$, we hope that it remains a frame when $a_{n}$ or $b_{n}$ has some small perturbation. For a regular Gabor frame $\left\{e^{i m b x} g(x-n a): m, n \in \mathbb{Z}\right\}$, it was shown in $\underline{6}$, 7, 15, 29, 30, that for certain window functions $g$, if $\left|\mu_{m, n}-n a\right|$ and $\left|\lambda_{m, n}-m b\right|$ are small enough, then $\left\{e^{i \lambda_{m, n} x} g\left(x-\mu_{m, n}\right): m, n \in \mathbb{Z}\right\}$ is also a frame.

In this paper, we study the stability of Gabor frames with arbitrary timefrequency parameters. We show that for any $g$ with $g(x), x g(x), x^{2} g(x) \in H^{2}$ and any sequence $\left\{\left(a_{n}, b_{n}\right)\right\}$, if $\left\{e^{i b_{n} x} g\left(x-a_{n}\right): n \in \mathbb{Z}\right\}$ is a frame, so is $\left\{e^{i b_{n}^{\prime} x} g\left(x-a_{n}^{\prime}\right)\right.$ : $n \in \mathbb{Z}\}$ provided $\left|a_{n}^{\prime}-a_{n}\right|$ and $\left|b_{n}^{\prime}-b_{n}\right|$ are small enough. The stability bounds are determined explicitly.

Notation and definition. The Fourier transform of $f \in L^{2}(\mathbb{R})$ is defined by $\hat{f}(\omega)=\int_{\mathbb{R}} f(x) e^{-i x \omega} d x$.

$H^{s}=\left\{f: \int_{-\infty}^{+\infty}\left(1+|\omega|^{2}\right)^{s}|\hat{f}(\omega)|^{2}<+\infty\right\}$ is the Sobolev space.

Let $\Gamma=\left\{\left(a_{n}, b_{n}\right): n \in \mathbb{Z}\right\} \subset \mathbb{R}^{2} . \Gamma$ is called $(p, q)$-uniformly discrete if $\mid a_{n}$ $a_{m} \mid \geq p$ or $\left|b_{n}-b_{m}\right| \geq q$ for any $m \neq n$. $\Gamma$ is called relatively uniformly discrete if it is a finite union of uniformly discrete sequences. $\Gamma$ is called $(a, b)$-dense if $\bigcup_{n \in \mathbb{Z}}\left[a_{n}-\frac{a}{2}, a_{n}+\frac{a}{2}\right] \times\left[b_{n}-\frac{b}{2}, b_{n}+\frac{b}{2}\right]=\mathbb{R}^{2}$.

We say a rectangle $E=\left[t_{1}, t_{2}\right] \times\left[\omega_{1}, \omega_{2}\right] \subset \mathbb{R}^{2}$ is of size $(a, b)$ if $t_{2}-t_{1}=a$ and $\omega_{2}-\omega_{1}=b .|E|$ denotes the Lebesgue measure of $E$. The norms of both $L^{2}(\mathbb{R})$ and $L^{2}\left(\mathbb{R}^{2}\right)$ are denoted by $\|\cdot\|$. The exact meaning can be seen by the context.

A family of functions $\left\{f_{j}: j \in J\right\}$ belonging to a separable Hilbert space $\mathcal{H}$ is said to be a frame if there exist positive constants $A$ and $B$ such that $A\|f\|^{2} \leq$ 
$\sum_{j \in J}\left|\left\langle f, f_{j}\right\rangle\right|^{2} \leq B\|f\|^{2}$ for every $f \in \mathcal{H}$. The numbers $A$ and $B$ are called the lower and upper frame bounds, respectively. If only the right-hand inequality holds, $\left\{f_{j}: j \in J\right\}$ is called a Bessel sequence.

\section{Irregular Gabor frames}

In this section, we study the construction of Gabor frames with arbitrary timefrequency parameters.

Lemma 2.1. (i) For any integer $n \geq 1, f \in H^{n}$ if and only if $f$ is $n-1$ times continuously differentiable, $f^{(n-1)}$ is locally absolutely continuous and $f, f^{\prime}, \cdots, f^{(n)}$ $\in L^{2}(\mathbb{R})$.

(ii) If $g(x), x g(x), \cdots, x^{n} g(x) \in L^{2}(\mathbb{R})$, then $\hat{g}$ is $n-1$ times continuously differentiable, $\hat{g}^{(n-1)}$ is locally absolutely continuous and $\hat{g}, \hat{g}^{\prime}, \cdots, \hat{g}^{(n)} \in L^{2}(\mathbb{R})$.

Proof. (i) This is a consequence of [3, Theorem 5.2].

(ii) Let $f(t)=\frac{1}{2 \pi} \hat{g}(-t)$. Then $\hat{f}(x)=g(x)$ and so $f \in H^{n}$. Now the conclusion follows from (i).

Remark. Although Sobolev spaces are involved in this paper, the differentiation is in the classical sense.

Lemma 2.2. Suppose that $f, g \in L^{2}(\mathbb{R})$. Then

(i) $\left\|\left(F_{g} f\right)(t, \omega)\right\|^{2}=2 \pi\|g(x)\|^{2} \cdot\|f(x)\|^{2}$.

(ii) $\left(F_{g} f\right)(t, \omega)=\frac{1}{2 \pi} e^{-i \omega t}\left(F_{\hat{g}} \hat{f}\right)(\omega,-t)$.

(iii) $\frac{\partial}{\partial t}\left(F_{g} f\right)(t, \omega) \stackrel{2 \pi}{=}-\left(F_{g^{\prime}} f\right)(t, \omega)$ if $g$ is differentiable and $g^{\prime} \in L^{2}(\mathbb{R})$.

Proof. The first two equalities are consequences of Parseval's identity. For the third one, it suffices to prove that

$$
\lim _{\triangle x \rightarrow 0}\left\|\frac{g(x+\triangle x)-g(x)}{\triangle x}-g^{\prime}(x)\right\|^{2}=\lim _{\triangle x \rightarrow 0} \frac{1}{2 \pi}\left\|\left(\frac{e^{i \triangle x \xi}-1}{\triangle x}-i \xi\right) \hat{g}(\xi)\right\|^{2}=0 .
$$

Since $\left|\frac{e^{i \Delta x \xi}-1}{\Delta x}\right|=\left|\frac{2 \sin \frac{\Delta x \xi}{\Delta x}}{\Delta x}\right| \leq|\xi|$ and $\xi \hat{g}(\xi)=-i \widehat{g^{\prime}}(\xi) \in L^{2}(\mathbb{R})$, the conclusion follows from the dominated convergence theorem.

Proposition 2.3 (Wirtinger's inequality [22]). If $f$ is differentiable on $[a, b], f, f^{\prime} \in$ $L^{2}[a, b]$ and $f(a) f(b)=0$, then

$$
\int_{a}^{b}|f(x)|^{2} d x \leq \frac{4}{\pi^{2}}(b-a)^{2} \int_{a}^{b}\left|f^{\prime}(x)\right|^{2} d x .
$$

The following lemma is an immediate consequence.

Lemma 2.4. If $f$ is differentiable on $[a, b], f, f^{\prime} \in L^{2}[a, b]$ and there is some $c \in[a, b]$ such that $f(c)=0$, then

$$
\int_{a}^{b}|f(x)|^{2} d x \leq \frac{4 \delta^{2}}{\pi^{2}} \int_{a}^{b}\left|f^{\prime}(x)\right|^{2} d x
$$

where $\delta=\max \{c-a, b-c\}$. 
Lemma 2.5. Let $\left\{E_{n}: n \in \mathbb{Z}\right\}$ be a sequence of measurable sets in $\mathbb{R}^{2}$ such that $\left|E_{n} \cap E_{m}\right|=0$, for $n \neq m$. Suppose that $u_{n}(t, \omega), v_{n}(t, \omega) \in L^{2}\left(E_{n}\right)$ and that $\sum_{n \in \mathbb{Z}}\left\|u_{n}(t, \omega)\right\|_{L^{2}\left(E_{n}\right)}^{2}$ and $\sum_{n \in \mathbb{Z}}\left\|v_{n}(t, \omega)\right\|_{L^{2}\left(E_{n}\right)}^{2}$ are finite. Then

$$
\begin{aligned}
& \left|\left(\sum_{n \in \mathbb{Z}}\left\|u_{n}(t, \omega)\right\|_{L^{2}\left(E_{n}\right)}^{2}\right)^{1 / 2}-\left(\sum_{n \in \mathbb{Z}}\left\|v_{n}(t, \omega)\right\|_{L^{2}\left(E_{n}\right)}^{2}\right)^{1 / 2}\right| \\
\leq & \left(\sum_{n \in \mathbb{Z}}\left\|u_{n}(t, \omega)-v_{n}(t, \omega)\right\|_{L^{2}\left(E_{n}\right)}^{2}\right)^{1 / 2} \\
\leq & \left(\sum_{n \in \mathbb{Z}}\left\|u_{n}(t, \omega)\right\|_{L^{2}\left(E_{n}\right)}^{2}\right)^{1 / 2}+\left(\sum_{n \in \mathbb{Z}}\left\|v_{n}(t, \omega)\right\|_{L^{2}\left(E_{n}\right)}^{2}\right)^{1 / 2} .
\end{aligned}
$$

Proof. By the triangle inequalities in $L^{2}\left(E_{n}\right)$ and $\ell^{2}(\mathbb{Z})$, we have

$$
\begin{aligned}
& \left(\sum_{n \in \mathbb{Z}}\left\|u_{n}(t, \omega)-v_{n}(t, \omega)\right\|_{L^{2}\left(E_{n}\right)}^{2}\right)^{1 / 2} \\
\geq & \left(\sum_{n \in \mathbb{Z}}\left|\left\|u_{n}(t, \omega)\right\|_{L^{2}\left(E_{n}\right)}-\left\|v_{n}(t, \omega)\right\|_{L^{2}\left(E_{n}\right)}\right|^{2}\right)^{1 / 2} \\
\geq & \left|\left(\sum_{n \in \mathbb{Z}}\left\|u_{n}(t, \omega)\right\|_{L^{2}\left(E_{n}\right)}^{2}\right)^{1 / 2}-\left(\sum_{n \in \mathbb{Z}}\left\|v_{n}(t, \omega)\right\|_{L^{2}\left(E_{n}\right)}^{2}\right)^{1 / 2}\right| .
\end{aligned}
$$

Similarly we can prove the second inequality.

Lemma 2.6. Suppose that $g(x), x g(x) \in H^{1}$. Let $a, b>0$ be such that

$$
\Delta:=\frac{2 a}{\pi}\left\|g^{\prime}(x)\right\|+\frac{2 b}{\pi}\|x g(x)\|+\frac{4 a b}{\pi^{2}}\left\|x g^{\prime}(x)\right\|<\|g(x)\| .
$$

Suppose that $E_{n}$ are rectangles with size no greater than $(a, b)$ such that $\bigcup_{n \in \mathbb{Z}} E_{n}=$ $\mathbb{R}^{2}$ and $\left|E_{m} \bigcap E_{n}\right|=0, n \neq m$. Then for any $\left(a_{n}, b_{n}\right) \in E_{n},\left\{\left|E_{n}\right|^{1 / 2} e^{i b_{n} x} g\left(x-a_{n}\right)\right.$ : $n \in \mathbb{Z}\}$ is a frame for $L^{2}(\mathbb{R})$ with bounds $2 \pi(\|g(x)\|-\Delta)^{2}$ and $2 \pi(\|g(x)\|+\Delta)^{2}$.

Proof. By Lemma 2.1] $g(x), g^{\prime}(x), x g(x), x g^{\prime}(x) \in L^{2}(\mathbb{R})$.

Put $E_{n}=\left[t_{n, 1}, t_{n, 2}\right] \times\left[\omega_{n, 1}, \omega_{n, 2}\right]$. Then $t_{n, 2}-t_{n, 1} \leq a$ and $\omega_{n, 2}-\omega_{n, 1} \leq b$. For any $f \in L^{2}(\mathbb{R})$, we derive from Lemma 2.2 that

$$
\begin{aligned}
& \sum_{n \in \mathbb{Z}} \iint_{E_{n}}\left|\left(F_{g} f\right)(t, \omega) e^{i \omega t}-\left(F_{g} f\right)\left(t, b_{n}\right) e^{i b_{n} t}\right|^{2} d \omega d t \\
= & \sum_{n \in \mathbb{Z}} \int_{t_{n, 1}}^{t_{n, 2}} d t \int_{\omega_{n, 1}}^{\omega_{n, 2}} \frac{1}{4 \pi^{2}}\left|\left(F_{\hat{g}} \hat{f}\right)(\omega,-t)-\left(F_{\hat{g}} \hat{f}\right)\left(b_{n},-t\right)\right|^{2} d \omega
\end{aligned}
$$




$$
\begin{aligned}
& \left.\leq \sum_{n \in \mathbb{Z}} \int_{t_{n, 1}}^{t_{n, 2}} d t \cdot \frac{4 b^{2}}{\pi^{2}} \int_{\omega_{n, 1}}^{\omega_{n, 2}} \frac{1}{4 \pi^{2}}\left|\left(F_{\hat{g}^{\prime}} \hat{f}\right)(\omega,-t)\right|^{2} d \omega \quad \text { (Lemma 2.4 }\right) \\
& =\sum_{n \in \mathbb{Z}} \frac{4 b^{2}}{\pi^{2}} \iint_{E_{n}}\left|\left(F_{\tilde{g}} f\right)(t, \omega)\right|^{2} d \omega d t \quad(\tilde{g}(x)=-i x g(x)) \\
& =\frac{4 b^{2}}{\pi^{2}}\left\|\left(F_{\tilde{g}} f\right)(t, \omega)\right\|^{2} \\
& =\frac{4 b^{2}}{\pi^{2}} \cdot 2 \pi\|x g(x)\|^{2}\|f(x)\|^{2} .
\end{aligned}
$$

Noting that

$$
\sum_{n \in \mathbb{Z}} \iint_{E_{n}}\left|\left(F_{g} f\right)(t, \omega) e^{i \omega t}\right|^{2} d \omega d t=\left\|\left(F_{g} f\right)(t, \omega)\right\|^{2}=2 \pi\|g(x)\|^{2}\|f(x)\|^{2},
$$

we see from Lemma 2.5 that

$$
\begin{aligned}
& \sum_{n \in \mathbb{Z}} \iint_{E_{n}}\left|\left(F_{g} f\right)\left(t, b_{n}\right)\right|^{2} d \omega d t \\
= & \sum_{n \in \mathbb{Z}} \iint_{E_{n}}\left|\left(F_{g} f\right)(t, \omega) e^{i \omega t}-\left(\left(F_{g} f\right)(t, \omega) e^{i \omega t}-\left(F_{g} f\right)\left(t, b_{n}\right) e^{i b_{n} t}\right)\right|^{2} d \omega d t \\
\leq & 2 \pi\left(\|g(x)\|+\frac{2 b}{\pi}\|x g(x)\|\right)^{2}\|f(x)\|^{2} .
\end{aligned}
$$

Hence

$$
\begin{aligned}
& \sum_{n \in \mathbb{Z}} \iint_{E_{n}}\left|\left(F_{g} f\right)\left(t, b_{n}\right) e^{i b_{n} t}-\left(F_{g} f\right)\left(a_{n}, b_{n}\right) e^{i b_{n} t}\right|^{2} d \omega d t \\
= & \sum_{n \in \mathbb{Z}} \int_{\omega_{n, 1}}^{\omega_{n, 2}} d \omega \int_{t_{n, 1}}^{t_{n, 2}}\left|\left(F_{g} f\right)\left(t, b_{n}\right)-\left(F_{g} f\right)\left(a_{n}, b_{n}\right)\right|^{2} d t \\
\leq & \sum_{n \in \mathbb{Z}} \frac{4 a^{2}}{\pi^{2}} \iint_{E_{n}}\left|\left(F_{g^{\prime}} f\right)\left(t, b_{n}\right)\right|^{2} d \omega d t \quad \text { (Lemma 2.4) } \\
\leq & \frac{4 a^{2}}{\pi^{2}} 2 \pi\left(\left\|g^{\prime}(x)\right\|+\frac{2 b}{\pi}\left\|x g^{\prime}(x)\right\|\right)^{2}\|f(x)\|^{2},
\end{aligned}
$$

where (2.3) is used in the last step.

Putting (2.1) and (2.4) together, we see from Lemma 2.5 that

$$
\begin{aligned}
& \sum_{n \in \mathbb{Z}} \iint_{E_{n}}\left|\left(F_{g} f\right)(t, \omega) e^{i \omega t}-\left(F_{g} f\right)\left(a_{n}, b_{n}\right) e^{i b_{n} t}\right|^{2} d \omega d t \\
\leq & 2 \pi\left(\frac{2 a}{\pi}\left\|g^{\prime}(x)\right\|+\frac{2 b}{\pi}\|x g(x)\|+\frac{4 a b}{\pi^{2}}\left\|x g^{\prime}(x)\right\|\right)^{2}\|f(x)\|^{2} \\
= & 2 \pi \Delta^{2}\|f(x)\|^{2} .
\end{aligned}
$$


Using (2.2) and Lemma 2.5 again, we get

$$
\begin{aligned}
& \sum_{n \in \mathbb{Z}}\left|E_{n}\right| \cdot\left|\left\langle f(x), e^{i b_{n} x} g\left(x-a_{n}\right)\right\rangle\right|^{2}=\sum_{n \in \mathbb{Z}} \iint_{E_{n}}\left|\left(F_{g} f\right)\left(a_{n}, b_{n}\right)\right|^{2} d \omega d t \\
= & \sum_{n \in \mathbb{Z}}\left\|\left(F_{g} f\right)(t, \omega) e^{i \omega t}-\left(\left(F_{g} f\right)(t, \omega) e^{i \omega t}-\left(F_{g} f\right)\left(a_{n}, b_{n}\right) e^{i b_{n} t}\right)\right\|_{L^{2}\left(E_{n}\right)}^{2} \\
\geq & 2 \pi(\|g(x)\|-\Delta)^{2}\|f(x)\|^{2} .
\end{aligned}
$$

Similarly we can prove that

$$
\sum_{n \in \mathbb{Z}}\left|E_{n}\right| \cdot\left|\left\langle f(x), e^{i b_{n} x} g\left(x-a_{n}\right)\right\rangle\right|^{2} \leq 2 \pi(\|g(x)\|+\Delta)^{2}\|f(x)\|^{2} .
$$

Now the conclusion follows.

Lemma 2.7. Suppose that $g(x), x g(x) \in H^{1}, p$ and $q$ are positive constants. Let

$$
M(g(x) ; p, q)=\|g(x)\|+\frac{p}{\pi}\left\|g^{\prime}(x)\right\|+\frac{q}{\pi}\|x g(x)\|+\frac{p q}{\pi^{2}}\left\|x g^{\prime}(x)\right\| .
$$

Then $\left\{e^{i b_{n} x} g\left(x-a_{n}\right): n \in \mathbb{Z}\right\}$ is a Bessel sequence in $L^{2}(\mathbb{R})$ with the bound $\frac{2 \pi}{p q} M(g(x) ; p, q)^{2}$ for any $(p, q)$-uniformly discrete sequence $\left\{\left(a_{n}, b_{n}\right): n \in \mathbb{Z}\right\}$.

Proof. Let $E_{n}=\left[a_{n}-\frac{p}{2}, a_{n}+\frac{p}{2}\right] \times\left[b_{n}-\frac{q}{2}, b_{n}+\frac{q}{2}\right]$. Then $\left|E_{n} \bigcap E_{m}\right|=0, m \neq n$. Similarly to Lemma 2.6$]$ we can prove that

$$
\begin{aligned}
& \sum_{n \in \mathbb{Z}} \iint_{E_{n}}\left|\left(F_{g} f\right)(t, \omega) e^{i \omega t}-\left(F_{g} f\right)\left(a_{n}, b_{n}\right) e^{i b_{n} t}\right|^{2} d \omega d t \\
\leq & 2 \pi\left(\frac{p}{\pi}\left\|g^{\prime}(x)\right\|+\frac{q}{\pi}\|x g(x)\|+\frac{p q}{\pi^{2}}\left\|x g^{\prime}(x)\right\|\right)^{2}\|f(x)\|^{2} .
\end{aligned}
$$

Hence

$$
\sum_{n \in \mathbb{Z}}\left|E_{n}\right| \cdot\left|\left\langle f(x), e^{i b_{n} x} g\left(x-a_{n}\right)\right\rangle\right|^{2} \leq 2 \pi M(g(x) ; p, q)^{2}\|f(x)\|^{2} .
$$

Since $\left|E_{n}\right|=p q$, the conclusion follows.

We are now ready to state the main results of this paper.

Theorem 2.8. Suppose that $g(x), x g(x) \in H^{1}$. Let $a, b>0$ be such that

$$
\Delta:=\frac{2 a}{\pi}\left\|g^{\prime}(x)\right\|+\frac{b}{\pi}\|x g(x)\|+\frac{2 a b}{\pi^{2}}\left\|x g^{\prime}(x)\right\|<\|g(x)\| .
$$

Then $\left\{e^{i b_{n} x} g\left(x-a_{n}\right): n \in \mathbb{Z}\right\}$ is a frame for $L^{2}(\mathbb{R})$ for any relatively uniformly discrete and $(a, b)$-dense sequence $\left\{\left(a_{n}, b_{n}\right): n \in \mathbb{Z}\right\}$.

Proof. Put $\Gamma=\left\{\left(a_{n}, b_{n}\right): n \in \mathbb{Z}\right\}$. We conclude that

$$
\Gamma \cap[t, t+a) \times[\omega, \omega+b) \neq \emptyset, \quad \forall(t, \omega) \in \mathbb{R}^{2} .
$$

Otherwise, there is some $\left(t_{0}, \omega_{0}\right) \in \mathbb{R}^{2}$ such that $\Gamma \cap\left[t_{0}, t_{0}+a\right) \times\left[\omega_{0}, \omega_{0}+b\right)=\emptyset$. Since $\Gamma$ is relatively uniformly discrete, there is some $\varepsilon>0$ such that $\Gamma \cap\left[t_{0}-\varepsilon\right.$, $\left.t_{0}+a\right) \times\left[\omega_{0}-\varepsilon, \omega_{0}+b\right)=\emptyset$. Hence $\left(t_{0}+\frac{a-\varepsilon}{2}, \omega_{0}+\frac{b-\varepsilon}{2}\right) \notin \bigcup_{n \in \mathbb{Z}}\left[a_{n}-\frac{a}{2}, a_{n}+\frac{a}{2}\right]$ $\times\left[b_{n}-\frac{b}{2}, b_{n}+\frac{b}{2}\right]=\mathbb{R}^{2}$, which is impossible.

For any $m \in \mathbb{Z}$, let $\Gamma_{m}=\left\{\left(a_{n}, b_{n}\right): m a \leq a_{n}<(m+1) a\right\}$. By (2.7), $\Gamma_{m}$ has infinitely many points and so we can write $\Gamma_{m}=\left\{\left(a_{n_{m, k}}, b_{n_{m, k}}\right): k \in \mathbb{Z}\right\}$. Without 
loss of generality, we can assume that $b_{n_{m, k}} \leq b_{n_{m, k+1}}$. Using (2.7) again, we have $\lim _{k \rightarrow \pm \infty} b_{n_{m, k}}= \pm \infty$ and $b_{n_{m, k+1}}-b_{n_{m, k}} \leq b$. Let

$$
E_{n_{m, k}}^{1}=[m a,(m+1) a] \times\left[\frac{b_{n_{m, k-1}}+b_{n_{m, k}}}{2}, b_{n_{m, k}}\right]
$$

and

$$
E_{n_{m, k}}^{2}=[m a,(m+1) a] \times\left[b_{n_{m, k}}, \frac{b_{n_{m, k}}+b_{n_{m, k+1}}}{2}\right] .
$$

Then $\left\{E_{n_{m, k}}^{j}: j=1\right.$ or $\left.2, m, k \in \mathbb{Z}\right\}$ meets Lemma 2.6 and $\left(a_{n_{m, k}}, b_{n_{m, k}}\right) \in$ $E_{n_{m, k}}^{1} \cap E_{n_{m, k}}^{2}$. By substituting $\frac{b}{2}$ for $b$ in Lemma 2.6. we have

$$
\begin{aligned}
& 2 \pi(\|g(x)\|-\Delta)^{2}\|f(x)\|^{2} \leq \sum_{m, k \in \mathbb{Z}}\left(\left|E_{n_{m, k}}^{1}\right|+\left|E_{n_{m, k}}^{2}\right|\right)\left|\left(F_{g} f\right)\left(a_{n_{m, k}}, b_{n_{m, k}}\right)\right|^{2} \\
& \leq \sum_{m, k \in \mathbb{Z}} a b\left|\left\langle f(x), e^{i b_{n_{m, k}} x} g\left(x-a_{n_{m, k}}\right)\right\rangle\right|^{2}=a b \sum_{n \in \mathbb{Z}}\left|\left\langle f(x), e^{i b_{n} x} g\left(x-a_{n}\right)\right\rangle\right|^{2} .
\end{aligned}
$$

On the other hand, since $\Gamma$ is relatively uniformly discrete, we can write $\Gamma=$ $\bigcup_{k=1}^{r} F_{k}$, where $F_{k}$ is a $\left(p_{k}, q_{k}\right)$-uniformly discrete sequence, $p_{k}, q_{k}>0,1 \leq k \leq r$, and $r$ is a positive integer. By Lemma 2.7.

$$
\begin{aligned}
\sum_{n \in \mathbb{Z}}\left|\left\langle f(x), e^{i b_{n} x} g\left(x-a_{n}\right)\right\rangle\right|^{2} & =\sum_{k=1}^{r} \sum_{\left(a_{n}, b_{n}\right) \in F_{k}}\left|\left\langle f(x), e^{i b_{n} x} g\left(x-a_{n}\right)\right\rangle\right|^{2} \\
& \leq\left(\sum_{k=1}^{r} \frac{2 \pi}{p_{k} q_{k}} M\left(g(x), p_{k}, q_{k}\right)^{2}\right)\|f(x)\|^{2} .
\end{aligned}
$$

This completes the proof.

Remark. The condition (2.6) can be replaced by

$$
\Delta:=\frac{a}{\pi}\left\|g^{\prime}(x)\right\|+\frac{2 b}{\pi}\|x g(x)\|+\frac{2 a b}{\pi^{2}}\left\|x g^{\prime}(x)\right\|<\|g(x)\|
$$

and the proof is similar to the one above.

\section{Stability of Gabor frames}

In this section, we study the stability of Gabor frames.

Noting that $\left(F_{g} f\right)\left(a_{n}+t_{0}, b_{n}+\omega_{0}\right)=e^{-i\left(b_{n}+\omega_{0}\right) t_{0}}\left\langle f\left(x+t_{0}\right) e^{-i \omega_{0} x}, e^{i b_{n} x} g\left(x-a_{n}\right)\right\rangle$, we have the following result.

Lemma 3.1. For any $g \in L^{2}(\mathbb{R})$, if $\left\{e^{i b_{n} x} g\left(x-a_{n}\right): n \in \mathbb{Z}\right\}$ is of a lower (upper) frame bound $A(B)$, so is $\left\{e^{i\left(b_{n}+\omega_{0}\right) x} g\left(x-a_{n}-t_{0}\right): n \in \mathbb{Z}\right\}$ for the same bound for any $t_{0}, \omega_{0} \in \mathbb{R}$.

Theorem 3.2. Suppose that $g(x), x g(x), x^{2} g(x) \in H^{2}$. Let $\left\{\left(a_{n}, b_{n}\right)\right\}$ be a $(p, q)$ uniformly discrete sequence and $\left\{e^{i b_{n} x} g\left(x-a_{n}\right): n \in \mathbb{Z}\right\}$ be a frame for $L^{2}(\mathbb{R})$ with bounds $A$ and $B$. Let $\delta, \eta>0$ be such that

$$
\Delta^{2}=\frac{2 \pi}{p q}\left(\frac{4 \delta}{\pi} M(x g(x) ; p, q)+\frac{4 \eta}{\pi} M\left(g^{\prime}(x) ; p, q\right)+\frac{16 \delta \eta}{\pi^{2}} M\left(x g^{\prime}(x) ; p, q\right)\right)^{2}<A .
$$

Then for any $\left(a_{n}^{\prime}, b_{n}^{\prime}\right)$ satisfying

$$
\left|a_{n}-a_{n}^{\prime}\right| \leq \eta \quad \text { and } \quad\left|b_{n}-b_{n}^{\prime}\right| \leq \delta
$$


$\left\{e^{i b_{n}^{\prime} x} g\left(x-a_{n}^{\prime}\right): n \in \mathbb{Z}\right\}$ is a frame for $L^{2}(\mathbb{R})$ with bounds $\left(A^{1 / 2}-\Delta\right)^{2}$ and $\left(B^{1 / 2}+\Delta\right)^{2}$.

Proof. It is easy to check that all of $M(x g(x) ; p, q), M\left(g^{\prime}(x) ; p, q\right)$ and $M\left(x g^{\prime}(x) ; p, q\right)$ are finite numbers, thanks to Lemma 2.1

For any $f \in L^{2}(\mathbb{R})$, by Lemma 2.2 and Lemma 2.4 we have

$$
\begin{aligned}
& \sum_{n \in \mathbb{Z}} \int_{-\eta}^{\eta} d t \int_{-\delta}^{\delta} \mid\left(F_{g} f\right)\left(a_{n}+t, b_{n}+\omega\right) e^{i\left(b_{n}+\omega\right)\left(a_{n}+t\right)} \\
& -\left.\left(F_{g} f\right)\left(a_{n}+t, b_{n}^{\prime}\right) e^{i b_{n}^{\prime}\left(a_{n}+t\right)}\right|^{2} d \omega \\
= & \sum_{n \in \mathbb{Z}} \int_{-\eta}^{\eta} d t \int_{-\delta}^{\delta} \frac{1}{4 \pi^{2}}\left|\left(F_{\hat{g}} \hat{f}\right)\left(b_{n}+\omega,-a_{n}-t\right)-\left(F_{\hat{g}} \hat{f}\right)\left(b_{n}^{\prime},-a_{n}-t\right)\right|^{2} d \omega \\
\leq & \sum_{n \in \mathbb{Z}} \frac{16 \delta^{2}}{\pi^{2}} \int_{-\eta}^{\eta} d t \int_{-\delta}^{\delta} \frac{1}{4 \pi^{2}}\left|\left(F_{\hat{g}^{\prime}} \hat{f}\right)\left(b_{n}+\omega,-a_{n}-t\right)\right|^{2} d \omega \\
= & \sum_{n \in \mathbb{Z}} \frac{16 \delta^{2}}{\pi^{2}} \int_{-\eta}^{\eta} d t \int_{-\delta}^{\delta}\left|\left(F_{\tilde{g}} f\right)\left(a_{n}+t, b_{n}+\omega\right)\right|^{2} d \omega \quad(\tilde{g}(x)=-i x g(x)) \\
\leq & \frac{16 \delta^{2}}{\pi^{2}} \cdot 4 \delta \eta \cdot \frac{2 \pi}{p q} M(x g(x) ; p, q)^{2}\|f(x)\|^{2},
\end{aligned}
$$

where Lemma 2.7] and Lemma 3.1 are used in the last step. Using Lemma 2.7 and Lemma 3.1 again, we get

$$
\sum_{n \in \mathbb{Z}} \int_{-\delta}^{\delta} d \omega \int_{-\eta}^{\eta}\left|\left(F_{g} f\right)\left(a_{n}+t, b_{n}+\omega\right)\right|^{2} d t \leq 4 \delta \eta \cdot \frac{2 \pi}{p q} M(g(x) ; p, q)^{2}\|f(x)\|^{2} .
$$

It follows from Lemma 2.5 that

$$
\begin{aligned}
& \sum_{n \in \mathbb{Z}} \int_{-\delta}^{\delta} d \omega \int_{-\eta}^{\eta}\left|\left(F_{g} f\right)\left(a_{n}+t, b_{n}^{\prime}\right)\right|^{2} d t \\
\leq & 4 \delta \eta \cdot \frac{2 \pi}{p q}\left(M(g(x) ; p, q)+\frac{4 \delta}{\pi} M(x g(x) ; p, q)\right)^{2}\|f(x)\|^{2} .
\end{aligned}
$$

Hence

$$
\begin{aligned}
& \sum_{n \in \mathbb{Z}} \int_{-\delta}^{\delta} d \omega \int_{-\eta}^{\eta}\left|\left(F_{g} f\right)\left(a_{n}+t, b_{n}^{\prime}\right) e^{i b_{n}^{\prime}\left(a_{n}+t\right)}-\left(F_{g} f\right)\left(a_{n}^{\prime}, b_{n}^{\prime}\right) e^{i b_{n}^{\prime}\left(a_{n}+t\right)}\right|^{2} d t \\
= & \sum_{n \in \mathbb{Z}} \int_{-\delta}^{\delta} d \omega \int_{-\eta}^{\eta}\left|\left(F_{g} f\right)\left(a_{n}+t, b_{n}^{\prime}\right)-\left(F_{g} f\right)\left(a_{n}^{\prime}, b_{n}^{\prime}\right)\right|^{2} d t \\
\leq & \sum_{n \in \mathbb{Z}} \frac{16 \eta^{2}}{\pi^{2}} \int_{-\delta}^{\delta} d \omega \int_{-\eta}^{\eta}\left|\left(F_{g^{\prime}} f\right)\left(a_{n}+t, b_{n}^{\prime}\right)\right|^{2} d t \\
\leq & \frac{16 \eta^{2}}{\pi^{2}} \cdot 4 \delta \eta \cdot \frac{2 \pi}{p q}\left(M\left(g^{\prime}(x) ; p, q\right)+\frac{4 \delta}{\pi} M\left(x g^{\prime}(x) ; p, q\right)\right)^{2}\|f(x)\|^{2} .
\end{aligned}
$$


By (3.1) and Lemma 2.5 we have

$$
\begin{aligned}
\sum_{n \in \mathbb{Z}} \int_{-\delta}^{\delta} d \omega & \int_{-\eta}^{\eta} \mid\left(F_{g} f\right)\left(a_{n}+t, b_{n}+\omega\right) e^{i\left(b_{n}+\omega\right)\left(a_{n}+t\right)} \\
& -\left.\left(F_{g} f\right)\left(a_{n}^{\prime}, b_{n}^{\prime}\right) e^{i b_{n}^{\prime}\left(a_{n}+t\right)}\right|^{2} d t \leq 4 \delta \eta \Delta^{2}\|f(x)\|^{2} .
\end{aligned}
$$

Using Lemma 3.1 again, we get

$$
\begin{aligned}
4 \delta \eta A\|f(x)\|^{2} & \leq \sum_{n \in \mathbb{Z}} \int_{-\delta}^{\delta} d \omega \int_{-\eta}^{\eta}\left|\left(F_{g} f\right)\left(a_{n}+t, b_{n}+\omega\right)\right|^{2} d t \\
& \leq 4 \delta \eta B\|f(x)\|^{2} .
\end{aligned}
$$

By Lemma 2.5 we have

$$
\begin{aligned}
\left(A^{1 / 2}-\Delta\right)^{2}\|f(x)\|^{2} & \leq \sum_{n \in \mathbb{Z}}\left|\left(F_{g} f\right)\left(a_{n}^{\prime}, b_{n}^{\prime}\right)\right|^{2} \\
& \leq\left(B^{1 / 2}+\Delta\right)^{2}\|f(x)\|^{2} .
\end{aligned}
$$

This completes the proof.

Since $\left\{\left(a_{n}, b_{n}\right): n \in \mathbb{Z}\right\}$ is the finite union of uniformly discrete sequences whenever $\left\{e^{i b_{n} x} g\left(x-a_{n}\right): n \in \mathbb{Z}\right\}$ is a frame [9], the following theorem can be proved similarly.

Theorem 3.3. Let $g$ be defined as in Theorem 3.2. Suppose $\left\{e^{i b_{n} x} g\left(x-a_{n}\right): n \in\right.$ $\mathbb{Z}\}$ is a frame for $L^{2}(\mathbb{R})$. Then there exist two positive constants $\delta$ and $\eta$ such that $\left\{e^{i b_{n}^{\prime} x} g\left(x-a_{n}^{\prime}\right): n \in \mathbb{Z}\right\}$ is a frame whenever $\left|a_{n}-a_{n}^{\prime}\right| \leq \eta$ and $\left|b_{n}-b_{n}^{\prime}\right| \leq \delta$.

Remark. 1. The stability bounds $\delta$ and $\eta$ can be determined explicitly as in Theorem 3.2. By [9, Theorem 3.1], there exist some $r \geq 1$ and $p_{k}, q_{k}>0,1 \leq k \leq r$, such that $\left\{\left(a_{n}, b_{n}\right): n \in \mathbb{Z}\right\}=\bigcup_{k=1}^{r}\left\{\left(a_{k, n}, b_{k, n}\right): n \in \Lambda_{k}\right\}, \Lambda_{k} \subset \mathbb{Z}$ and $\left\{\left(a_{k, n}, b_{k, n}\right)\right.$ : $\left.n \in \Lambda_{k}\right\}$ is $\left(p_{k}, q_{k}\right)$-uniformly discrete. Let

$$
\begin{aligned}
\Delta^{\prime 2}=\sum_{k=1}^{r} & \frac{2 \pi}{p_{k} q_{k}}\left(\frac{4 \delta}{\pi} M\left(x g(x) ; p_{k}, q_{k}\right)\right. \\
& \left.+\frac{4 \eta}{\pi} M\left(g^{\prime}(x) ; p_{k}, q_{k}\right)+\frac{16 \delta \eta}{\pi^{2}} M\left(x g^{\prime}(x) ; p_{k}, q_{k}\right)\right)^{2} .
\end{aligned}
$$

Then $\Delta^{\prime}<A^{1 / 2}$ meets the requirement of Theorem 3.3 and the frame bounds are $\left(A^{1 / 2}-\Delta^{\prime}\right)^{2}$ and $\left(B^{1 / 2}+\Delta^{\prime}\right)^{2}$.

2. It is easy to see that $g$ meets Theorem 3.2 and 3.3 if $g$ or $\hat{g}$ is 2 times continuously differentiable and compactly supported.

\section{ACKNowledgement}

We thank the referee very much for very valuable and elaborate suggestions which helped us to improve this paper. 


\section{REFERENCES}

[1] H.Bacry, A.Grossmann, and J.Zak, Proofs of the completeness of lattice states in the kqrepresentation, Phys. Rev., B12(1975), 1118-1120.

[2] V.Bargmann, P.Butera, L.Girardello and J.R.Klauder, On the completeness of coherent states, Rep. Math. Phys., 2(1971), 221-228. MR 44:7860

[3] J.J.Benedetto, C.Heil, and D.Walnut, Differentiation and the Balian-Low theorem, J. Fourier Anal. Appl., 1(1995), 355-402. MR 96f:42002

[4] P.G.Casazza and O.Christensen, Weyl-Heisenberg frames for subspaces of $L^{2}(\mathbb{R})$, Proc. Amer. Math. Soc., 129(2001), 145-154. MR 2001h:42046

[5] O.Christensen, Atomic decomposition via projective group representations, Rocky Mountain J. Math., 26(1996), 1289-1312. MR 98h:43004

[6] O.Christensen, Moment problems and stability results for frames with applications to irregular sampling and Gabor frames, Appl. Comp. Harmonic Anal., 3(1996), 82-86. MR 97f:44007

[7] O.Christensen, Perturbation of operators and applications to frame theory, J. Four. Anal. Appl., 3(1997), 543 - 557. MR 98j:47028

[8] O.Christensen, Frames, Riesz bases, and discrete Gabor/wavelet expansions, Bulletin (New series) of Amer. Math. Soc., 38(2001), 273-291. MR 2002c:42040

[9] O.Christensen, B.Deng, and C.Heil, Density of Gabor frames, Appl. Comput. Harmon. Anal., 7(1999), 292-304. MR 2000j:42043

[10] C.K.Chui and X.L.Shi, Inequalities of Littlewood-Paley type for frames and wavelets, SIAM J. Math. Anal., 24(1993), 263-277. MR 94d:42039

[11] I.Daubechies, The wavelet transform, time-frequency localization and signal analysis, IEEE Trans. Inform. Theory, 36(1990), 961-1005. MR 91e:42038

[12] I.Daubechies, Ten Lectures on Wavelets, SIAM Philadelphia, 1992. MR 93e:42045

[13] I.Daubechies and A.Grossmann, Frames of entire functions in the Bargmann space, Comm. Pure Appl. Math., 41(1988), 151-164. MR 89e:46028

[14] I.Daubechies, H.Landau and Z.Landau, Gabor time-frequency lattices and the Wexler-Raz identity, J. Fourier Anal. Appl., 4(1995), 437-478. MR 96i:42021

[15] S.Favier and R.Zalik, On the stability of frames and Riesz bases, Appl. and Comp. Harm. Anal., 2(1995), 160-173. MR 96e:42030

[16] H.G.Feichtinger and K.Gröchenig, Banach spaces related to integrable group representations and their atomic decomposition, J. funct. Anal., 86(1989), 307-340. MR 91g:43011

[17] H.G.Feichtinger and A.J.E.M.Janssen, Validity of $W H$-frame bound conditions depends on lattice parameters, Appl. Comput. Harmon. Anal., 8(2000), 104-112. MR 2000j:42044

[18] H.G.Feichtinger and T.Strohmer, Eds., Gabor Analysis and Algorithms: Theory and Applications, Birkhäuser, Boston, 1997. MR 98h:42001

[19] D.Gabor, Theory of communications, J. Inst. Elect. Eng.(London), 93(1943), 429-457.

[20] K. Gröchenig, Describing functions: atomic decompositions versus frames, Mh. Math., 112(1991), 1-41. MR 92m:42035

[21] K. Gröchenig, Irregular sampling of wavelet and short-time Fourier transforms, Constr. Approx., 9(1993), 283-297. MR 94m:42077

[22] G.Hardy, J.E.Littlewood, and G.Pólya, Inequalities, 2nd Ed.,Cambridge: Cambridge Univ. Press, 1952. MR 13:727e

[23] C.Heil and D.Walnut, Continuous and discrete wavelet transforms, SIAM Review, 31(1989), 628-666. MR 91c:42032

[24] Y.Lyubarskii, Frames in the Bargmann space of entire functions, in Entire and subharmonic functions, vol 11 of the series Advanced in Soviet Mathematics, B.Ya. Levin, ed., Springerverlag, Berlin, 1992. MR 93k:30036

[25] A.M.Perelomov, On the completeness of a system of coherent states, Teor. Mat. Fiz., 6(1971), 213-224. MR 57:15050

[26] J.Ramanathan and T.Steger, Incompleteness of sparse coherent states, Appl. Comput. Harmon. Anal., 2(1995), 148-153. MR 96b:81049

[27] A.Ron and Z.Shen, Weyl-Heisenberg frames and Riesz bases in $L^{2}\left(\mathbb{R}^{d}\right)$, Duke Math. J., 89(1997), 148-153. MR 98i:42013

[28] K.Seip and R.Wallstén, Density theorems for sampling and interpolation in the BargmannFock space II, J. Reine Angew. Math., 429(1992), 107-113. MR 93g:46026b 
[29] W.Sun and X.Zhou, On the stability of Gabor frames, Advances in Applied Mathematics, 26(2001), 181-191. MR 2002c: 42043

[30] W.Sun and X.Zhou, Irregular wavelet/Gabor frames, Appl. Comput. Harmon. Anal., 13 (2002), 63-76.

[31] R.Young, An Introduction to Non-Harmonic Fourier Series, Academic Press, New York, 1980. MR 81m:42027

Department of Mathematics, Nankai University, Tianjin 300071, People's Republic OF CHINA

E-mail address: sunwch@nankai.edu.cn

Department of Mathematics, Nankai University, Tianjin 300071, People's Republic OF CHINA

E-mail address: xwzhou@nankai.edu.cn 